\title{
PARTITIONS OF UNITY AND A CLOSED EMBEDDING THEOREM FOR $\left(C^{p}, b^{*}\right)$-MANIFOLDS
}

\author{
BY \\ RICHARD E. HEISEY( $\left.{ }^{1}\right)$
}

\begin{abstract}
Many manifolds of fiber bundle sections possess a natural atlas $\left\{\left(U_{\alpha}, \phi_{\alpha}\right)\right\}$ such that the transition maps $\phi_{\beta} \phi_{\alpha}^{-1}$, in addition to being smooth, are continuous with respect to the bounded weak topology of the model. In this paper we formalize the idea of such manifolds by defining $\left(C^{D}, b^{*}\right)$-manifolds, $\left(C^{p}, b^{*}\right)$-morphisms, etc. We then show that these manifolds admit $\left(C^{p}, b^{*}\right)$ partitions of unity subordinate to certain open covers and that they can be embedded as closed $\left(C^{p}, b^{*}\right)$-submanifolds of their model. A corollary of our work is that for any Banach space $B$, the conjugate space $B^{*}$ admits smooth partitions of unity subordinate to covers by sets open in the bounded weak-* topology.
\end{abstract}

0. Introduction. In $\S 3$ of [10] Richard Palais discusses an additional structure possessed by certain manifolds of fiber bundle sections. (For example, the manifold of $L_{k}^{p}$ cross sections of a fiber bundle over a smooth compact $n$ dimensional manifold $M, p>1$ and $k>n / p$. See [5] for this and other examples.) This additional structure can be described by saying that the manifold possesses a natural atlas $\left\{\left(U_{\alpha}, \phi_{\alpha}\right)\right\}$ such that the transition maps $\phi_{\beta} \phi_{\alpha}^{-1}$, in addition to being smooth, are continuous with respect to the bounded weak topology of the model. (The bounded weak topology on a Banach space $B$ is the finest topology agreeing with the weak topology on bounded sets.) In this paper we formalize the idea of such manifolds by defining $\left(C^{p}, b^{*}\right)$-manifolds, $\left(C^{p}, b^{*}\right)$ morphisms, etc. We then show that these manifolds admit $\left(C^{p}, b^{*}\right)$-partitions of unity subordinate to certain open covers and that they can be embedded as closed $\left(C^{p}, b^{*}\right)$-submanifolds of their model.

A corollary of our work is that given any Banach space $B$, the conjugate space $B^{*}$ admits smooth partitions of unity subordinate to covers by sets open in the bounded weak-* topology. This is in contrast to the fact that there are conjugate spaces known not to admit smooth functions of bounded support (see §II,

Presented to the Society, August 30,1972 under the title A closed embedding theorem; received by the editors October 19, 1972 and, in revised form, February 21, 1974.

AMS (MOS) subject classifications (1970). Primary 58B10, 54E55; Secondary 57D40. Key words and phrases. Manifolds of sections, bounded weak topology, partitions of unity, closed embedding.

(1) This research was supported by a NASA Fellowship (1970-1971) and a Rotary Foundation Fellowship (1971-1972). It constitutes part of the author's Ph. D. thesis at Cornell University. 
Theorem II. 2 and the paragraph following it).

The basic definitions and the precise statements of our main results are contained in $\S$ II.

Throughout this paper we work with the bounded weak-* topology on the conjugate, $B^{*}$, of a Banach space $B$ rather than the bounded weak topology on $B$. By doing so we are able to avoid the hypothesis of reflexivity by appealing to Alaoglu's theorem. (Alaoglu's theorem [3, p. 424] states that given any Banach space $B$, the closed unit ball in $B^{*}$ with its weak-* topology is compact.) We then obtain as corollaries theorems about manifolds which are modelled on reflexive Banach spaces and whose transition maps respect the bounded weak topology. (See the remark at the end of §II.) The reader interested in only this (reflexive) case may well want to read this paper by replacing " $B$ " by " $B$ " and "bounded weak-* topology" by "bounded weak topology". Then reflexivity (rather than Alaoglu's theorem) will give the weak compactness of bounded sets. Propositions III. 2 and III. 3 may then be omitted, and the rest of our definitions and proofs go through with only minor changes.

The idea of considering manifolds modelled on conjugate spaces and bounded weak-* topologies originates with Richard Graff. Since first submitting this paper for publication the author has received a copy of Graff's thesis [5] which investigates manifolds modelled on conjugate spaces. Graff has independently obtained a strong partition of unity result in the case that $B$ is separable [5, Corollary 4.22 , p. 118]. (Related to this see also [5, Theorem 4.21, p. 117] and [5, Example 2.40, p. 64].) Note, however, that our partition of unity result, Theorem II. 1, has no separability restriction. Graff has also obtained, independently, a proof of our Proposition III. 1(e) [5, Proposition 2.7, p. 42].

The author wishes to thank his thesis advisor, David W. Henderson, for suggesting this research and for continually giving help and encouragement. Also, the author wishes to thank David Elworthy, who made several very valuable suggestions resulting in the improvement of this paper. It should be noted that our proof of Theorem II. 3, and especially our introduction of a $\left(\tau^{*}, b^{*}\right)$-Banach completion in $\S \mathrm{V}$, is patterned after the work of N. H. Kuiper and B. TerpstraKeppler in [7]. Finally, the author wishes to thank the referee for several helpful suggestions. In particular, the referee showed how to simplify the proof of Theorem V. 5.

I. Notation. Throughout this paper $B, E$, and $F$ will denote real Banach spaces. The norm on any Banach space will be denoted by \|\| . Given a Banach space $B, B^{*}$ denotes the conjugate of $B$. On $B^{*}$ we consider three topologies: $\tau_{B}^{*}$, the metric topology (induced by the norm); $w_{B}^{*}$, the weak-* topology (sometimes called the $B$-topology of $B^{*}$, see $\left.[3, \mathrm{p} .420]\right)$; and $b_{B}^{*}$, the bounded weak-* 
topology (sometimes called the bounded $B$-topology for $B^{*}$, see [3, p. 427]). Recall that $b_{B}^{*}$ is the finest topology on $B^{*}$ that agrees with $w_{B}^{*}$ on (norm) bounded sets of $B^{*}$. When no confusion will result we suppress the subscripts and write $\tau^{*}, w^{*}$, and $b^{*}$. By $B^{*}\left(\tau^{*}\right)$ (resp. $B^{*}\left(w^{*}\right), B^{*}\left(b^{*}\right)$ ), we indicate $B^{*}$ with its $\tau^{*}$ (resp. $w^{*}, b^{*}$ ) topology. For any real number $r>0$, let $B_{r}^{*}=\left\{x^{*} \in B^{*} \mid\left\|x^{*}\right\| \leqslant\right.$ $r$ \}. We use the notation $B=E \oplus F$ to mean that $B$ is the topological direct sum of the closed linear subspaces $E$ and $F$. As usual $R$ denotes the space of real numbers.

II. Basic definitions and summary of results. Let $M$ be a set, $-1 \leqslant p \leqslant \infty$. In order to have the following definitions cover the cases $p=-1$ and $p=0$, we regard a $C^{-1}$-diffeomorphism as a set bijection, a $C^{0}$-diffeomorphism as a homeomorphism, etc. (Thus, our $\left(C^{-1}, b^{*}\right)$-manifolds will be topological manifolds carrying only a $b^{*}$ topology, and our $\left(C^{0}, b^{*}\right)$-manifolds will be topological manifolds carrying both a $b^{*}$ and a metric topology.) $\mathrm{A}\left(C^{p}, b^{*}\right)$-atlas for $M$ is a collection of pairs $\left\{\left(U_{i}, \phi_{i}\right) \mid i \in I\right\}, I$ some indexing set, such that (1) each $U_{i} \subset M$, (2) $M=\bigcup_{i \in I} U_{i}$, (3) for every $i, j \in I$ such that $U_{i} \cap U_{j} \neq \varnothing, \phi_{i}\left(U_{i} \cap U_{j}\right)$ is open in $B_{i}^{*}\left(b^{*}\right)$ for some Banach space $B_{i}$, and (4) for every $i, j$ with $U_{i} \cap U_{j} \neq$ $\varnothing, \phi_{j} \phi_{i}^{-1}: \phi_{i}\left(U_{i} \cap U_{j}\right) \rightarrow \phi_{j}\left(U_{i} \cap U_{j}\right)$ is a $C^{p}$-diffeomorphism w.r.t. the metric topologies and a homeomorphism w.r.t. the $b^{*}$ topologies of $B_{i}^{*}$ and $B_{j}^{*}$. Any such pair $\left(U_{i}, \phi_{i}\right)$ will be called a $\left(C^{p}, b^{*}\right)$-chart on $M$. As usual, two $\left(C^{p}, b^{*}\right)$ atlases will be called compatible if their union is again a $\left(C^{\rho}, b^{*}\right)$-atlas for $M$. "Compatibility" is then an equivalence relation on the family of $\left(C^{p}, b^{*}\right)$-atlases for $M$. An equivalence class of $\left(C^{p}, b^{*}\right)$-atlases for $M$ will be called a $\left(C^{p}, b^{*}\right)$ manifold structure on $M$, and $M$ equipped with a $\left(C^{p}, b^{*}\right)$-manifold structure will be called a $\left(C^{p}, b^{*}\right)$-manifold. Note that a $\left(C^{p}, b^{*}\right)$-atlas determines two topologies on $M$. One is defined by requiring each $U_{i}$ to be open and each $\phi_{i}: U_{i} \rightarrow$ $\phi_{i}\left(U_{i}\right) \subset B_{i}^{*}\left(\tau^{*}\right)$ to be a homeomorphism for each $i$. The other is defined by requiring each $U_{i}$ to be open and each $\phi_{i}: U_{i} \rightarrow \phi_{i}\left(U_{i}\right) \subset B_{i}^{*}\left(b^{*}\right)$ to be a homeomorphism. When regarding $M$ with the first (resp. second) of these topologies we write $M\left(\tau^{*}\right)$ (resp. $M\left(b^{*}\right)$ ). It is clear that equivalent atlases induce the same topologies, so that a $\left(C^{p}, b^{*}\right)$-manifold has well defined $\tau^{*}$ and $b^{*}$ topologies.

If a $\left(C^{p}, b^{*}\right)$-manifold $M$ has a $\left(C^{p}, b^{*}\right)$-atlas $\left\{\left(U_{i}, \phi_{i}\right)\right\}$ such that all the $\phi_{i}\left(U_{i}\right)$ lie in the same conjugate space, say $B^{*}$, then we say $M$ is a $\left(C^{p}, b^{*}\right)$ manifold modelled on $B^{*}$. Note that $B^{*}$ is naturally a $\left(C^{p}, b^{*}\right)$-manifold modelled on $B^{*},-1 \leqslant p \leqslant \infty$.

Let $M, N$ be two $\left(C^{p}, b^{*}\right)$-manifolds. A map $f: M \rightarrow N$ is a $\left(C^{p}, b^{*}\right)$ morphism if $\forall x \in M$ there is a $\left(C^{p}, b^{*}\right)$-chart $(U, \phi)$ about $x$ and a $\left(C^{p}, b^{*}\right)$ chart $(V, \psi)$ about $f(x)$ such that $f(U) \subset V$ and $\psi f \phi^{-1}$ is a $C^{p}$-morphism in the 
$\tau^{*}$-topologies and a continuous map in the $b^{*}$-topologies. We obtain a category of $\left(C^{p}, b^{*}\right)$-manifolds and $\left(C^{p}, b^{*}\right)$-morphisms. If $f: M \rightarrow N$ has an inverse in this category it will be called a $\left(C^{p}, b^{*}\right)$-isomorphism.

Let $M$ be a $\left(C^{p}, b^{*}\right)$-manifold, and let $A \subset M$ be such that for every $x \in A$ there is a $\left(C^{p}, b^{*}\right)$-chart $(U, \phi)$ such that $\phi(U)=U_{i} \oplus U_{2} \subset B^{*}$ and $\phi(U \cap A)=$ $U_{1}$ where $U_{i}$ is open in $E_{i}^{*}, i=1,2$, and $B=E_{1} \oplus E_{2}$. (Here we have identified $B^{*}$ with $E_{1}^{*} \oplus E_{2}^{*}$. We use the hypothesis $B=E_{1} \oplus E_{2}$ to insure that the natural map $E_{1}^{*} \oplus E_{2}^{*} \rightarrow E_{1}^{*} \times E_{2}^{*}$ is a homeomorphism in the $b^{*}$, as well as $\tau^{*}$, topologies. See Proposition III. 3.) Regarding $\phi / U \cap A$ as a map into $E_{1}^{*}$, the charts $(U \cap A$, $\phi / U \cap A)$ induce a $\left(C^{p}, b^{*}\right)$-manifold structure on $A$. We call $A$ with this structure a $\left(C^{p}, b^{*}\right)$-submanifold of $M$. If $A$ is also closed in $M\left(b^{*}\right)$ we call $A$ a closed $\left(C^{p}, b^{*}\right)$-submanifold of $M$.

If $f: M \rightarrow N$ is a $\left(C^{p}, b^{*}\right)$-morphism and $x \in M$ we say $f$ is a split $\left(C^{p}, b^{*}\right)$ embedding at $x$ if there is a neighborhood $W$ of $x$ in $M\left(b^{*}\right)$ such that $f / W: W \rightarrow$ $f(W)$ is a $\left(C^{p}, b^{*}\right)$-isomorphism onto a $\left(C^{p}, b^{*}\right)$-submanifold of $N$. (Note that our definition of $\left(C^{p}, b^{*}\right)$-submanifold incorporates a strong splitting requirement. See [4, p. 767].) We say $f$ is a local split $\left(C^{p}, b^{*}\right)$ embedding if it is a split $\left(C^{p}, b^{*}\right)$-embedding at each point of $M$. If a local split $\left(C^{p}, b^{*}\right)$-embedding gives a $\left(C^{p}, b^{*}\right)$-isomorphism onto a [closed] $\left(C^{p}, b^{*}\right)$-submanifold of $N$ we call it a [closed] split $\left(C^{p}, b^{*}\right)$-embedding.

A $\left(C^{p}, b^{*}\right)$-partition of unity for a $\left(C^{p}, b^{*}\right)$-manifold $M$ is a topological partition of unity $\left\{\lambda_{\alpha}: M \rightarrow R\right\}$ for $M\left(b^{*}\right)$ such that each $\lambda_{\alpha}$ is a $\left(C^{p}, b^{*}\right)$ morphism. (Here we have identified $R$ with $R^{*}$.)

We are now ready to state our main results.

THEOREM II. 1. Let $M$ be $a\left(C^{p}, b^{*}\right)$-manifold such that $M\left(b^{*}\right)$ is paracompact. Then $M$ admits $a\left(C^{p}, b^{*}\right)$-partition of unity subordinate to any $b^{*}$-open cover, $-1 \leqslant p \leqslant \infty$.

The proof of Theorem II. 1 is given in §IV. Note, as an immediate corollary to Theorem II. 1 , that the $\left(C^{p}, b^{*}\right)$-manifold $M$ admits a $C^{p}$-partition of unity subordinate to any $b^{*}$-open cover. In Proposition III. 1(d) we show that for any Banach space $B, B^{*}\left(b^{*}\right)$ is paracompact. Thus we obtain:

Theorem II. 2 (Corollary of II. 1). For any Banach space $B, B^{*}$ admits smooth partitions of unity subordinate to $b^{*}$-open covers.

Thus, for example, $l_{1}=c_{0}^{*}$ admits smooth partitions of unity subordinate to $b^{*}$-open covers. (Here $l_{1}$ is the space of all real sequences $\left\{x_{i}\right\}$ with $\left\|\left\{x_{i}\right\}\right\|=$ $\Sigma_{i}\left|x_{i}\right|<\infty$, and $c_{0}$ is the space of all real sequences $\left\{c_{i}\right\}$ converging to zero with 
$\left\|\left\{c_{i}\right\}\right\|=\sup _{i}\left\{\left|c_{i}\right|\right\}$. The isomorphism $l_{1} \stackrel{\Phi}{\longrightarrow} c_{0}^{*}$ is given by $\Phi\left(\left\{x_{i}\right\}\right)\left(\left\{c_{i}\right\}\right)=$ $\Sigma c_{i} x_{i}$.) This is in contrast to the fact shown by Bonic and Frampton in [1] that there is no nontrivial differentiable map $f: l_{1} \rightarrow R$ with bounded support. (That there is no such continuously differentiable map $f$ was shown by Kurzweil in [8].)

THEOREM II. 3. Let $M$ be $a\left(C^{p}, b^{*}\right)$-manifold modelled on $B^{*}$ such that $M\left(b^{*}\right)$ is regular and Lindelöf. If there is a closed linear split embedding $B \oplus$ $B \rightarrow B$, then there is a closed split $\left(C^{p}, b^{*}\right)$ embedding $M \stackrel{f}{\rightarrow} B^{*},-1 \leqslant p \leqslant \infty$.

Theorem II. 3 is a corollary of the more general Theorem V. 5 stated and proved in $\S \mathrm{V}$.

If $B$ is separable we may weaken the hypothesis " $M\left(b^{*}\right)$ is regular and Lindelöf" occurring in Theorem II. 3. Assuming $B$ is separable, each bounded set in $B^{*}\left(b^{*}\right)$ is metrizable [3, Theorem 1, p. 426]. Thus, if $O$ is open in $B^{*}\left(b^{*}\right)$, $O \cap B_{n}^{*}\left(b^{*}\right)$ is an $F_{\sigma}$ set, $n=1,2, \cdots$. Since $B_{n}^{*}\left(b^{*}\right)$ is compact (Alaoglu's theorem), $O \cap B_{n}^{*}\left(b^{*}\right)$ is thus the countable union of compact sets. It follows that $O$ is the countable union of compact sets and therefore Lindelöf. But, as shown in Proposition III. 4, if every open subset of the model is Lindelöf, then each component of the paracompact manifold $M$ is Lindelöf. We have proven the following theorem.

Theorem II. 4 (Corollary of II. 3). Assume B is separable. Let $M$ be $a\left(C^{p}, b^{*}\right)$-manifold modelled on $B^{*}$ such that $M\left(b^{*}\right)$ is paracompact with at most countably many components. If there is a closed linear split embedding $B \oplus B$ $\rightarrow B$, then there is a closed spit $\left(C^{p}, b^{*}\right)$ embedding $M \stackrel{f}{\rightarrow} B^{*},-1 \leqslant p \leqslant \infty$.

REMARK. If $B$ is a Banach space, let $b$ denote the bounded weak topology on $B$. Define $\left(C^{p}, b\right)$-manifolds in analogy with $\left(C^{p}, b^{*}\right)$-manifolds. If $B$ is reflexive, then the natural isomorphism $B \rightarrow B^{* *}$ identifies the $b$ topology on $B$ with the $b^{*}$ (i.e. bounded $B^{*}$ ) topology on $B^{* *}$. Hence our theorems yield as special cases analogous theorems about $\left(C^{p}, b\right)$-manifolds modelled on reflexive Banach spaces.

III. Some preliminaries. First we recall the following theorem due to Banach and Dieudonné [2]; for the theorem in the form we state it see $[3, p$. 427].

THEOREM (BANACH-DiEudonNÉ). A fundamental system of neighborhoods of the origin for the $b^{*}$-topology of $B^{*}$ consists of the sets $\left\{x^{*} \in B^{*}|| x^{*}(a) \mid<\right.$ $1, a \in A\}$ where $A$ is a sequence of elements of $B$ converging to zero in the norm topology. 
Since translation is a homeomorphism on $B^{*}\left(b^{*}\right)$ it follows that if $x_{0} \in B$, a basis for $B^{*}\left(b^{*}\right)$ at $x_{0}^{*}$ is the collection of sets $\left\{x^{*} \in B^{*}||\left(x^{*}-x_{0}^{*}\right)(a) \mid<1\right.$, $a \in A\}$ where $A$ is a sequence in $B$ converging to zero in the norm topology.

Proposition III. 1. (a) $B^{*}\left(b^{*}\right)$ is a locally convex topological vector space.

(b) Any open set in $B^{*}\left(b^{*}\right)$ is unbounded if $B$ is infinite dimensional.

(c) If $U=\left\{x^{*} \in B^{*}|| x^{*}(a) \mid<1, a \in A\right\}$ where $A$ is any subset of $B$, and if $\bar{U}$ is the closure of $U$ in $B^{*}\left(b^{*}\right)$, then $\bar{U}=\left\{x^{*} \in B^{*}|| x^{*}(a) \mid \leqslant 1, a \in A\right\}$.

(d) $B^{*}\left(b^{*}\right)$ is regular, Lindelöf, and paracompact.

(e) $B^{*}\left(b^{*}\right)$ is not first countable if $B$ is infinite dimensional.

Proof of III. 1. (a) is an immediate corollary of the Banach-Dieudonné theorem. For (b), let $V=\left\{x^{*} \in B^{*}|| x^{*}(a) \mid<1, a \in A\right\}$, where $A$ is a sequence in $B$ converging to the origin in the metric topology, be a basic open neighborhood of the origin in $B^{*}\left(b^{*}\right)$, and let $r>0$. Set $A^{\prime}=\{a \in A \mid\|a\| \geqslant 1 / r\}$. Then $A^{\prime}$ is finite, and so, if $B$ is infinite dimensional, the closed linear span of $A$ is not all of $B$. From the Hahn-Banach theorem it follows [11, Theorem 5.19] that there is a linear functional $y^{*} \in B^{*}$ such that $y^{*}\left(A^{\prime}\right)=0$ and $\left\|y^{*}\right\|=r$. Then $y^{*} \in V$ and we have shown that $V$ contains elements of arbitrary norm.

For (c), note that the linear functional determined by each $a \in A\left(x^{*} \rightarrow\right.$ $\left.x^{*}(a), x^{*} \in B^{*}\right)$ is continuous on $B^{*}\left(b^{*}\right)$. Thus the set $\left\{x^{*} \in B|| x^{*}(a) \mid \leqslant 1\right\}$ is closed in $B^{*}\left(b^{*}\right)$. This set is exactly the closure of $U$ as each $x^{*} \in B^{*}$ is the limit in the $b^{*}$ topology of a sequence $\left\{t_{n} x^{*}\right\},\left\{t_{n}\right\}$ being any sequence in $(0,1)$ converging to 1 .

To see that $B^{*}\left(b^{*}\right)$ is regular, let $U=\left\{x^{*} \in B^{*}|| x^{*}(a) \mid<1, a \in A\right\}$ be a basic open neighborhood of zero. Setting $1 / 2 U=\left\{x^{*} \in B^{*}|| x^{*}(a) \mid<1 / 2, a \in A\right\}$, from (c) we have $0 \in 1 / 2 U \subset \overline{1 / 2 U} \subset U$, and regularity follows. $B^{*}\left(b^{*}\right)$ is Lindelöf since it is the countable union of compact subspaces (Alaoglu theorem). By Morita's theorem [9, Theorem 10] any regular Lindelöf space is paracompact. This completes the proof of (d).

We prove (e), by contradiction. Suppose $B^{*}\left(b^{*}\right)$ were first countable. For every $n$, let $S_{n}^{*}=\left\{x^{*} \in B^{*} \mid\left\|x^{*}\right\|=n\right\}$, and let $\bar{S}_{n}^{*}$ be the closure of $S_{n}^{*}$ in $B^{*}\left(b^{*}\right)$. From (b) it follows (we are assuming $B$ is infinite dimensional) that $0 \in \bar{S}_{1}^{*}$. Hence by our supposition there is a sequence $\left\{x_{i}^{*} \mid i=1,2, \cdots\right\} \subset S_{1}^{*}$ such that $x_{i}^{*} \stackrel{b^{*}}{\longrightarrow} 0$ (i.e. $\left\{x_{i}^{*}\right\}$ converges to 0 in the $b^{*}$ topology). Similarly, for each $i$ there is a sequence $\left\{x_{i, k}^{*} \mid k=1,2, \cdots\right\} \subset S_{i+1}^{*}$ converging to $x_{i}^{*}$ in the $b^{*}$ topology. Let $A=\left\{x_{i, k}^{*} \mid i, k=1,2, \cdots\right\}$. Then the $b^{*}$ closure of $A$ contains 0 . By our supposition of first countability there is a sequence $\left\{a_{j} \mid j=1,2, \cdots\right\}$ $\subset A$ such that $a_{j} \stackrel{b^{*}}{\longrightarrow} 0$. But then clearly also $a_{j} \stackrel{w^{*}}{\longrightarrow} 0$ so $\left\{a_{j}\right\}$ is bounded. 
Hence, for some integer $n \geqslant 2,\left\{a_{j}\right\} \cap S_{n}^{*}$ is an infinite sequence convergent to 0 . This is a contradiction since $\left\{a_{j}\right\} \cap S_{n}^{*}$ must converge to $x_{n}^{*}$.

For convenience we include the following proposition which is probably well known.

Proposition III. 2. Let $\lambda: B^{*} \rightarrow E^{*}$ be a linear map. The following assertions are equivalent and imply that $\lambda: B^{*}\left(\tau^{*}\right) \rightarrow E^{*}\left(\tau^{*}\right)$ is continuous:

(a) $\lambda: B^{*}\left(w^{*}\right) \rightarrow E^{*}\left(w^{*}\right)$ is continuous.

(b) $\lambda: B^{*}\left(b^{*}\right) \rightarrow E^{*}\left(b^{*}\right)$ is continuous.

(c) $\lambda$ is the adjoint of a continuous (w.r.t the metric topologies) linear map $\gamma: E \rightarrow B$.

Proof OF III. 2. (a) implies (b): By [3, Corollary 3, p. 424] the compact sets in $B^{*}\left(w^{*}\right)$ are the closed sets in $B^{*}\left(w^{*}\right)$ that are bounded in $B^{*}\left(\tau^{*}\right)$. Thus, if $\lambda$ is $w^{*}$-continuous (i.e. continuous w.r.t. the $w^{*}$-topologies) $\lambda$ carries bounded sets to bounded sets. It follows at once that $\lambda$ is $b^{*}$-continuous.

(b) implies (c): We know [3, Theorem 6, p. 428 and Theorem 9, p. 421] that the conjugate of $B^{*}\left(b^{*}\right)$ is $B$, and the metric topology on $B$ considered as the conjugate of $B^{*}\left(b^{*}\right)$ is obviously the norm topology $\tau$. If $\lambda: B^{*}\left(b^{*}\right) \rightarrow$ $E^{*}\left(b^{*}\right)$ is continuous, then, as in (a) implies (b), $\lambda$ is bounded and hence $\tau^{*}$. continuous. Thus, the adjoint map $\lambda^{*}: E \rightarrow B$ is $\tau$-continuous [3, Lemma 2, p. 478]. Clearly $\lambda$ is the adjoint of $\lambda^{*}$.

(c) implies (a): Easy, standard, and left to the reader.

If $B=E \oplus F$ we identify $\left\{x^{*} \in B^{*} \mid x^{*}(F)=0\right\}$ with $E^{*}$ and $\left\{x^{*} \in B^{*} \mid\right.$ $x^{*}(E)=0$ \} with $F^{*}$. Using this identification we have:

Proposition III. 3. If $B=E \oplus F$, then $B^{*}=E^{*} \oplus F^{*}$, and the natural linear map $E^{*} \oplus F^{*} \rightarrow E^{*} \times F^{*}$ given by $x^{*}+y^{*} \rightarrow\left(x^{*}, y^{*}\right)$ is a homeomorphism w.r.t. the $w^{*}$ and $b^{*}$ (as well as $\tau^{*}$ ) topologies.

Proof of III. 3. Let $i: E \rightarrow B, j: F \rightarrow B$ be the inclusions and $p: B \rightarrow$ $E, q: B \rightarrow F$ the (continuous) projections given by the direct sum $B=E \oplus F$. Then

$$
p \circ i=I_{E}, \quad q \circ j=I_{F}, \quad \text { and } i \circ p+j \circ q=I_{B} .
$$

By III. 2 the adjoint maps $i^{*}, j^{*}, p^{*}, q^{*}$ are all continuous w.r.t. the $\tau^{*}$, $w^{*}$, and $b^{*}$ topologies. Clearly, also,

$$
i^{*} \circ p^{*}=I_{E^{*}}, \quad j^{*} \circ q^{*}=I_{F^{*}}, \quad p^{*} \circ i^{*}+q^{*} \circ j^{*}=I_{B^{*}}
$$

The proposition follows.

Finally, we establish the following proposition, which was used in §II to 
establish Theorem II. 4. The technique used in the proof, as noted in [6, Lemma $1.1]$, is essentially due to Bill Cutler.

PROPOSITION III. 4. Let $M$ be a connected (Hausdorff) paracompact manifold modelled on the topological vector space $F$. If each open subset of $F$ is Lindelöf, then $M$ is Lindelöf. If also $F$ is separable, then so also is $M$.

Proof of III. 4. Let $\left\{U_{\alpha} \mid \alpha \in \mathcal{Q}\right\}$ be a locally-finite open cover of $X$ by Lindelöf sets. Then for each $\alpha, U_{\alpha}$ is regular and Lindelöf and hence paracompact (Morita, [9, Theorem 10]). Let $\left\{O_{\beta} \mid \beta \in \mathfrak{B}\right\}$ be any given open cover of $X$. For each $\gamma \in \mathcal{Q}$ consider the cover $\left\{\mathcal{O}_{\beta} \cap U_{\gamma} \mid \beta \in \mathfrak{B}\right\}$ of $U_{\gamma}$. Since $U_{\gamma}$ is paracompact and Lindelöf, there is a countable, locally-finite open refinement $\left\{V_{i}^{\gamma} \mid\right.$ $i=1,2, \cdots\}$ of $\left\{O_{\beta} \cap U_{\gamma} \mid \beta \in \mathscr{B}\right\}$ with each $V \gamma$ meeting only finitely many $U_{\alpha}$ 's. Define a chain on $X$ to be a finite collection of open sets $\left\{W_{i} \mid i=1, \cdots\right.$, $n$ \} such that $W_{i} \cap W_{i+1} \neq \varnothing, i=1, \cdots, n-1 . W_{i}$ is called the ith link of the chain, and the chain joins $x$ to $y$ if $x \in W_{1}$ and $y \in W_{n}$. Given $x \in X$, there are at most countably many chains starting at $x$ (i.e., $x \in W_{1}$ ) and made up of links from $\left\{V_{i}^{\alpha} \mid \alpha \in \mathscr{A}, i=1,2, \cdots\right\}$. This is because there are only countably many choices for the $i$ th link. The set of points that can be joined to $x$ by such chains is open and closed in connected $X$, and is therefore all of $X$. Thus there is such a chain having any given $V_{i}^{\alpha}$ as a link. This in turn implies that $\left\{V_{i}^{\alpha} \mid \alpha \in \mathcal{U}, i=\right.$ $1,2, \cdots\}$ must be countable. Choosing an $O_{\beta}$ containing each $V_{i}^{\alpha}$ gives the required countable subcover of $\left\{O_{\beta} \mid \beta \in \mathfrak{B}\right\}$.

The final assertion of III. 4 follows since the countable union of separable spaces is separable.

IV. Proof of Theorem II. 1. From the Banach-Dieudonne theorem (see $\S$ III) sets of the form $\left\{x^{*} \in B^{*}||\left(x^{*}-x_{0}^{*}\right)\left(x_{i}\right) \mid<\alpha_{i}, i=1,2,3, \cdots\right\}$, where $x_{i} \in B,\left\|x_{i}\right\|=1$ and $\alpha_{i} \rightarrow \infty$, form a basis at $x_{0}^{*} \in B^{*}\left(b^{*}\right)$.

LEMMA IV. 1. Let $U=\left\{x^{*} \in B^{*}||\left(x^{*}-x_{0}^{*}\right)\left(x_{i}\right) \mid<\alpha_{i}, i=1,2,3, \cdots\right\}$, where $x_{i} \in B,\left\|x_{i}\right\|=1$ and $\alpha_{i} \rightarrow \infty$, be a basic open neighborhood of $x_{0}^{*}$ in $B^{*}\left(b^{*}\right)$. Let $U(2)=\left\{x^{*} \in B^{*}||\left(x^{*}-x_{0}^{*}\right)\left(x_{i}\right) \mid<2 \alpha_{i}, i=1,2,3, \cdots\right\}$. Then there exists $a\left(C^{\infty}, b^{*}\right)$-morphism $\phi: B^{*} \rightarrow[0,1] \subset R$ such that $\phi\left(B^{*} \backslash U(2)\right)=$ 0 and $\phi / \bar{U}=1$, where $\bar{U}$ is the closure of $U$ in $B^{*}\left(b^{*}\right)$.

Proof OF IV. 1. We may assume $x_{0}^{*}=0$. It follows from III. 1(c) that $\bar{U}=\left\{x^{*} \in B^{*}|| x^{*}\left(x_{i}\right) \mid \leqslant \alpha_{i}, i=1,2,3, \cdots\right\}$. For each $i$, let $\widetilde{\phi}_{i}: R \rightarrow[0,1]$ be a $C^{\infty}$ map such that $\widetilde{\phi}_{i} /\left[-\alpha_{i}, \alpha_{i}\right]=1$ and $\widetilde{\phi}_{i} /\left(R \backslash\left(-2 \alpha_{i}, 2 \alpha_{i}\right)\right)=0$. Define $\phi_{i}: B^{*} \rightarrow[0,1]$ by $\phi_{i}=\widetilde{\phi}_{i} \circ \hat{x}_{i}$ where $\hat{x}_{i}: B^{*}\left(w^{*}\right) \rightarrow R$ is the continuous linear functional $\hat{x}_{i}\left(y^{*}\right)=y^{*}\left(x_{i}\right)$. Then $\phi_{i}: B^{*} \rightarrow R$ is a $\left(C^{\infty}, b^{*}\right)$-morphism. Define 
$\phi: B^{*} \rightarrow[0,1]$ by $\phi=\Pi_{i=1}^{\infty} \phi_{i}$. Since $\alpha_{i} \rightarrow \infty$, given any $r>0, \phi$ restricted to $B_{r}^{*}$ reduces to a finite product. It follows easily that $\phi$ is a $\left(C^{\infty}, b^{*}\right)$-morphism satisfying the conditions of the lemma.

Proposition IV. 2. Let $A$ be a closed subset of $B^{*}\left(b^{*}\right)$ and $U$ an open subset of $B^{*}\left(b^{*}\right)$ such that $A \subset U$. Then there is a $\left(C^{\infty}, b^{*}\right)$-morphism $\lambda: B^{*} \rightarrow$ $[0,1] \subset R$ such that $\lambda / A=1$ and $\lambda /\left(B^{*} \backslash U\right)=0$.

Proof of IV. 2. For each integer $n \geqslant 1$, let $A_{n}=A \cap B_{n}^{*}$. Since $A_{1}\left(b^{*}\right)$ is compact (Alaoglu theorem), there are finitely many basic open sets in $B^{*}\left(b^{*}\right)$, say $V_{1,1}, \cdots, V_{1, k_{1}}$, covering $A_{1}$ such that $V_{1, i}(2) \subset U, i=1, \cdots, k_{1}$. For every $x^{*} \in A_{2} \backslash B_{1}^{*}$ let $V_{2, x}$ be a basic open neighborhood of $x^{*}$ in $B^{*}\left(b^{*}\right)$ such that $V_{2, x}$ (2) $\subset U \backslash B_{1}^{*}$. Then $V_{1,1}, \cdots, V_{1, k_{1}},\left\{V_{2, x} \mid x^{*} \in A_{2} \backslash B_{1}^{*}\right\}$ cover the $b^{*}$ compact $A_{2}$, so we may select a finite subcover, say $V_{1,1}, \cdots, V_{1, k_{1}}$, $V_{2,1}, \cdots, V_{2, k_{2}}$. Continuing inductively, we obtain a sequence $V_{1,1}, \cdots, V_{1, k_{1}}$, $V_{2,1}, \cdots, V_{i, 1}, \cdots, V_{i, k_{i}}, \cdots$ of basic $b^{*}$ open sets covering $A$ such that each $V_{i, j}(2) \subset U \backslash B_{i-1}^{*}, i>1$. Fix $i$. Define $\phi_{i}: B^{*} \rightarrow[0,1]$ as follows. By Lemma IV. 1 for each $j=1, \cdots, k_{i}$ there is a $\left(C^{\infty}, b^{*}\right)$-morphism $\phi_{i, j}: B^{*} \rightarrow[0,1]$ such that $\phi_{i, j} / V_{i, j}=0$ and $\phi_{i, j} / B^{*}-V_{i, j}(2)=1$. Let $\phi_{i}=\Pi_{j=1}^{k_{i}} \phi_{i, j}$. Then let $\phi=\prod_{i=1}^{\infty} \phi_{i}$. Then, since $\phi / B_{n}^{*}=\prod_{i=1}^{n} \phi_{i}$, it follows easily that $\phi$ is a $\left(C^{\infty}, b^{*}\right)$ morphism. Clearly, also, $\phi / A=0$ and $\phi /\left(B^{*} \backslash U\right)=1$. Defining $\lambda\left(x^{*}\right)=1-\phi\left(x^{*}\right)$ gives the required $\left(C^{\infty}, b^{*}\right)$-morphism.

Proof of Theorem II. 1. Having established Proposition IV. 2, Theorem II. 1 follows in rather standard fashion. We omit the proof.

\section{Proof of Theorem II. 3.}

Proposition V. 1. Let $f: M \rightarrow N$ be a local split $\left(C^{p}, b^{*}\right)$-embedding, $-1 \leqslant p \leqslant \infty$, which is also a topological embedding $M\left(b^{*}\right) \rightarrow N\left(b^{*}\right)$. Then $f$ is a split $\left(C^{p}, b^{*}\right)$ embedding.

Proof of V. 1. We must show that $f(M)$ is a $\left(C^{p}, b^{*}\right)$-submanifold of $N$ and that $f^{-1}: f(M) \rightarrow M$ is a $\left(C^{p}, b^{*}\right)$-morphism. Let $m \in M$. Since $f$ is a local split $\left(C^{p}, b^{*}\right)$-embedding there is a $\left(C^{p}, b^{*}\right)$-chart $(U, \phi)$ at $m$ such that $f: U \rightarrow$ $f(U)$ is a $\left(C^{p}, b^{*}\right)$-isomorphism onto a $\left(C^{p}, b^{*}\right)$-submanifold of $N$. By the defining property of $\left(C^{p}, b^{*}\right)$-submanifold there is a $\left(C^{p}, b^{*}\right)$-chart $(V, \psi)$ about $f(m)$ in $N$ such that $\psi(V)=V_{1} \oplus V_{2} \subset B^{*}, \psi(V \cap f(U))=V_{1}$, where $V_{i}$ is open in $E_{i}^{*}\left(b^{*}\right), i=1,2$, and $B=E_{1} \oplus E_{2}$. Since $f(U)$ is open in $f(M)$ regarded as a subspace of $N\left(b^{*}\right), f(U)=f(M) \cap W$ for some $b^{*}$-open set $W$ in $N$. Certainly we can require $V \subset W$. Then $\psi(V \cap f(M))=\psi(V \cap f(U))=V_{1}$. It follows that $f(M)$ is a $\left(C^{p}, b^{*}\right)$-submanifold of $N$. 
To see that $f^{-1}$ is a $\left(C^{p}, b^{*}\right)$-morphism let $\left(V^{\prime}, \psi^{\prime}\right)=\left(V \cap f(M), \psi_{1} / V \cap\right.$ $f(M)$ ) where $\psi_{1}$ is the composite of $\psi$ and the projection $V_{1} \oplus V_{2} \rightarrow V_{1}$. Then $\left(V^{\prime}, \psi^{\prime}\right)$ is a $\left(C^{p}, b^{*}\right)$-chart at $f(m)$ in $f(U)$, and hence (since $f: U \rightarrow f(U)$ is a $\left(C^{p}, b^{*}\right)$-isomorphism), $\phi f \psi^{\prime-1}$ is a $\left(C^{p}, b^{*}\right)$-morphism. But $\left(V, \psi^{\prime}\right)$ is also a $\left(C^{p}, b^{*}\right)$-chart at $f(m)$ in $f(M)$, so this shows $f^{-1}: f(M) \rightarrow M$ is a $\left(C^{p}, b^{*}\right)$. morphism at $f(m)$.

Definition V. 2 (CF. [7]). Let $\Sigma B^{*}$ be the countable direct limit of $B^{*}$. Again we have two topologies on $\Sigma B^{*}$ : the $\tau^{*}$ topology obtained by regarding $\Sigma B^{*}$ as $\Sigma B^{*}\left(\tau^{*}\right)$ and the $b^{*}$ topology obtained by regarding $\Sigma B^{*}$ as $\Sigma B^{*}\left(b^{*}\right)$. For every $n \geqslant 1, \Sigma B^{*}=\left(B^{*}\right)^{n} \oplus_{n} B^{*}$ where $\left(B^{*}\right)^{n}=\Sigma_{i=1}^{n} B^{*}$ and ${ }_{n} B^{*}=$ $\left\{x=\left\{x_{i}^{*}\right\} \in \Sigma B^{*} \mid x_{i}^{*}=0, i \leqslant n\right\}$. Let $E$ be a Banach space. A continuous linear injection $\chi: \Sigma B^{*}\left(b^{*}\right) \rightarrow E^{*}\left(b^{*}\right)$ will be called a $\left(\tau^{*}, b^{*}\right)$-Banach completion of $\Sigma B^{*}$ if, for every $n$,

(a) $\chi /\left(B^{*}\left(b^{*}\right)\right)^{n}$ is a closed $b^{*}$-embedding.

(b) there is a $b^{*}$-closed linear subspace ${ }_{n} E^{*}$ of $E^{*}$ such that $E^{*}=\chi\left(\left(B^{*}\right)^{n}\right)$ $\oplus_{n} E^{*}$ where $\chi\left(\left(B^{*}\right)^{n}\right) \oplus_{n} E^{*}$ is of the form $F_{1, n}^{*} \oplus F_{2, n}^{*}$ with $E=F_{1, n} \oplus F_{2, n}$, $\chi\left({ }_{n} B^{*}\right) \subset{ }_{n} E^{*}$ and $(n+1) E^{*} \subset{ }_{n} E^{*}$.

Note that by III. 2, if $\chi$ is a $\left(\tau^{*}, b^{*}\right)$-Banach completion, then $\chi /\left(B^{*}\right)^{n}$ is also an embedding w.r.t. the metric topologies.

Proposition V. 3. If there is a closed linear split embedding (metric topologies) $B \oplus B \rightarrow B$, then there is $a\left(\tau^{*}, b^{*}\right)$-Banach completion $\Sigma B^{*} \rightarrow B^{*}$.

Proof of V. 3. The lemma of [7] asserts that our hypothesis implies the existence of a continuous linear injection $\rho: \Sigma B \rightarrow B$ (metric topologies) such that for $n \geqslant 1, \rho / B^{n}$ is a closed embedding and, writing $B=B^{n} \oplus_{n} B$, there is a closed linear subspace of $B,{ }_{n} E$, such that $B=\rho\left(B^{n}\right) \oplus_{n} E,{ }_{(n+1)} E \subset{ }_{n} E$, and $\rho\left({ }_{n} B\right) \subset_{n} E$. For $n \geqslant 1$, let $\rho_{n}=\rho / B^{n}$. Define $\chi: \Sigma B^{*} \rightarrow B^{*}$ by $\chi /\left(B^{*}\right)^{n}=$ $\left(\rho_{n}^{-1}\right)^{*}$. (Here we identify $\left(B^{*}\right)^{n}$ with $\left(B^{n}\right)^{*}$.) One checks that $\chi$ is well defined and gives the required $\left(\tau^{*}, b^{*}\right)$-Banach completion.

Definition V. 4. Let $M$ be a $\left(C^{p}, b^{*}\right)$-manifold, $-1 \leqslant p \leqslant \infty$. A closed $\left(C^{p}, b^{*}\right)$-embedding $f: M \rightarrow \Sigma B^{*}$ is a closed topological embedding $f: M\left(b^{*}\right) \rightarrow$ $\Sigma B^{*}\left(b^{*}\right)$ such that for each $x \in M$ there is a neighborhood $U$ of $x$ in $M\left(b^{*}\right)$ and an integer $n(U, n$ depending on $x)$ such that $f(U) \subset\left(B^{*}\right)^{n}$ and $f: U \rightarrow\left(B^{*}\right)^{n}$ is a split $\left(C^{p}, b^{*}\right)$-embedding as defined in $\S I I$. Here we identify $\left(B^{*}\right)^{n}$ with $\left(B^{n}\right)^{*}$ and regard $\left(B^{*}\right)^{n}$ as a $\left(C^{p}, b^{*}\right)$-manifold modelled on $\left(B^{n}\right)^{*}$.

TheOREM V. 5. Let $M$ be $a\left(C^{p}, b^{*}\right)$-manifold modelled on $B^{*}$ such that $M\left(b^{*}\right)$ is regular and Lindelöf. Then there is a closed $\left(C^{p}, b^{*}\right)$ embedding $f: M$ $\rightarrow \Sigma B^{*}$ such that if $\chi: \Sigma B^{*} \rightarrow E^{*}$ is $a\left(\tau^{*}, b^{*}\right)$-Banach completion, then 
$\chi \circ f: M \rightarrow E^{*}$ is a closed split $\left(C^{p}, b^{*}\right)$-embedding, $-1 \leqslant p \leqslant \infty$.

Combined with Proposition V. 3, Theorem V. 5 immediately implies Theorem II. 3. Note that the proof of Theorem II. 4 given in §II shows that here, too, if $B$ is separable we may replace the hypothesis " $M\left(b^{*}\right)$ is regular and Lindelöf" by " $M\left(b^{*}\right)$ is paracompact with at most countably many components".

Proof OF V. 5. In this proof closures of sets will always be taken in the $b^{*}$ topology.

By Morita's theorem [9, Theorem 10], $M\left(b^{*}\right)$ being regular and Lindelöf implies $M\left(b^{*}\right)$ is also paracompact. For each $m \in M$, let $\left(U_{m}, \phi_{m}\right)$ be a $\left(C^{p}, b^{*}\right)$ chart with $m \in U_{m}$. Choose $G_{m}$ open in $B^{*}\left(b^{*}\right)$ such that

$$
\phi_{m}(m) \in G_{m} \subset \bar{G}_{m} \subset \phi_{m}\left(U_{m}\right) .
$$

Note that for any set $U \subset \phi_{m}^{-1}\left(G_{m}\right)$ we have $\phi_{m}(\bar{U})=\overline{\phi_{m}(U)}$. Using this together with the fact that $M^{*}\left(b^{*}\right)$ is paracompact and Lindelöf, one obtains a countable $\left(C^{p}, b^{*}\right)$-atlas $\left\{\left(U_{i}, \phi_{i}\right) \mid i=1,2, \cdots\right\}$ for $M$ such that $\left\{U_{i}\right\}$ is a locally-finite collection of nonempty sets, and each $\phi_{i}$ extends to a closed $b^{*}$-embedding $\overline{\phi_{i}}$ : $\overline{U_{i}} \rightarrow \overline{\phi_{i}\left(U_{i}\right)}$. Let $\left\{W_{i}\right\},\left\{O_{i}\right\},\left\{V_{i}\right\}$ be precise $b^{*}$-open refinements of $\left\{U_{i}\right\}$ such that for each $i$,

$$
\varnothing \neq W_{i} \subset \bar{w}_{i} \subset O_{i} \subset \bar{O}_{i} \subset V_{i} \subset \bar{V}_{i} \subset U_{i} .
$$

By IV. 2 there is for each $i$ a $\left(C^{p}, b^{*}\right)$-morphism $\lambda_{i}^{\prime}: B^{*} \rightarrow[0,1]$ such that $\lambda_{i}^{\prime} / \phi_{i}\left(\bar{W}_{i}\right)=1$ and $\lambda_{i}^{\prime} /\left(B^{*} \backslash \phi_{i}\left(O_{i}\right)\right)=0$. Define $\lambda_{i}: M \rightarrow[0,1]$ by $\lambda_{i} \mid U_{i}=$ $\lambda_{i}^{\prime} \circ \phi_{i}, \lambda_{i} /\left(M \backslash \bar{O}_{i}\right)=0$. Then $\lambda_{i}$ is a $\left(C^{p}, b^{*}\right)$-morphism with $\lambda_{i} / \bar{W}_{i}=1$ and $\lambda_{i} /\left(M \backslash O_{i}\right)=0$. Similarly, using the $V_{i}$, construct for each $i$ a $\left(C^{p}, b^{*}\right)$-morphism $\mu_{i}: M \rightarrow[0,1]$ such that $\mu_{i} / \mathcal{O}_{i}=1$ and $\mu_{i} /\left(M \backslash U_{i}\right)=0$. Fix a nonzero element $a^{*}$ of $B^{*}$. Define a $\left(C^{p}, b^{*}\right)$-morphism $\widetilde{\phi}_{i}: M \rightarrow B^{*} \times B^{*}$ by

$$
\widetilde{\phi}_{i}(m)=\left\{\begin{array}{l}
\left(\lambda_{i}(m) \phi_{i}(m), \lambda_{i}(m) \cdot a^{*}\right), \quad m \in U_{i}, \\
(0,0), \quad m \notin \bar{O}_{i} .
\end{array}\right.
$$

Since $\lambda_{i}=1$ on $W_{i}$, each $\widetilde{\phi}_{i}$ restricted to $W_{i}$ is a split $\left(C^{p}, b^{*}\right)$-embedding. Define $f: M \rightarrow \Sigma B^{*}$ by

$$
f(m)=\left(\sum_{i=1}^{\infty} i \cdot \mu_{i}(m) a^{*}, \widetilde{\phi}_{1}(m), \widetilde{\phi}_{2}(m), \cdots\right) .
$$

The local-finiteness of the $U_{i}$ insures that the sum is finite and that $f(m) \in \Sigma B^{*}$ (i.e. at most finitely many $\widetilde{\phi}_{i}(m)$ are nonzero for each $m$ ).

Let $m_{0} \in M$. Find an integer $j$ such that $m_{0} \in W_{j}$ and then a $b^{*}$ neighborhood $V$ of $m_{0}$ such that $V \subset W_{j}$ and $V$ intersects only $U_{1}, \cdots, U_{n}$ some $n$. 
Then for every $m \in V$,

$$
f(m)=\left(\sum_{i=1}^{\infty} i \mu_{i}(m) a^{*}, \widetilde{\phi}_{1}(m), \cdots, \widetilde{\phi}_{n}(m)\right) \in\left(B^{*}\right)^{2 n+1} .
$$

For convenience, define $f_{i}: V \rightarrow B^{*}, i=1, \cdots, 2 n+1$, such that

$$
f(m)=\left(\sum_{i=1}^{n} i \mu_{i}(m) a^{*}, \widetilde{\phi}_{1}(m), \cdots, \widetilde{\phi}_{n}(m)\right)=\left(f_{1}(m), \cdots, f_{2 n+1}(m)\right) .
$$

Note that $f_{2 j}(m)=\phi_{j}(m)$. Let $G=\Sigma_{i=1}^{2 n+1} X_{i}$ where $X_{i}=B^{*}, i \neq 2 j$, and $X_{2 j}=$ $\phi_{j}(V)$. Define $\psi: G \rightarrow G$ by $\psi\left(y_{1}, \cdots, y_{2 n+1}\right)=\left(z_{1}, \cdots, z_{2 n+1}\right)$ where $z_{i}=y_{i}-f_{i} \phi_{j}^{-1}\left(y_{2 j}\right), i \neq 2 j$ and $z_{2 j}=y_{2 j}$. Then $\psi$ is a $\left(C^{p}, b^{*}\right)$-isomorphism onto $G$, and $(G, \psi)$ is a $\left(C^{\phi}, b^{*}\right)$-chart at $f\left(m_{0}\right)$ in $\left(B^{*}\right)^{2 n+1}$. Clearly $\psi(f(V) \cap G)$ $=0 \times \cdots \times 0 \times \phi_{j}(V) \times 0 \times \cdots \times 0$, which shows $f(V)$ is a $\left(C^{p}, b^{*}\right)$-submanifold of $\left(B^{*}\right)^{2 n+1}$. The map $f(V) \rightarrow V$ given by $\left(y_{1}, \cdots, y_{2 n+1}\right) \rightarrow \phi_{j}^{-1}\left(y_{2 j}\right)$ is $f^{-1}$ and is clearly a $\left(C^{p}, b^{*}\right)$-morphism. Thus we have shown that each $m_{0} \in$ $M$ has a neighborhood $V$ in $M\left(b^{*}\right)$.such that $f(V) \subset\left(B^{*}\right)^{2 n+1}$, some $n$, and such that $f: V \rightarrow\left(B^{*}\right)^{2 n+1}$ is a split $\left(C^{p}, b^{*}\right)$-embedding.

To finish the proof that $f: M \rightarrow \Sigma B^{*}$ is a closed $\left(C^{p}, b^{*}\right)$-embedding it is only left to show that $f: M\left(b^{*}\right) \rightarrow \Sigma B^{*}\left(b^{*}\right)$ is a topological closed embedding. Clearly $f$ is injective. Let $A$ be closed in $M\left(b^{*}\right)$. Let $\left\{f\left(m_{\alpha}\right) \mid \alpha \in \mathscr{Q}\right\}$ be a net in $f(A)$ converging to $y$ in $\Sigma B^{*}\left(b^{*}\right)$. (We work with nets since the $b^{*}$ topology is generally not first countable, see III. 1(e).) Writing $y=\left(y_{1}, y_{2}, \cdots\right)$ we then must have $\left\{\Sigma_{i=1}^{\infty} i \mu_{i}\left(m_{\alpha}\right) a^{*} \mid \alpha \in \mathfrak{Q}\right\}$ converging to $y_{1}$ in $B^{*}\left(b^{*}\right)$. Hence, for some $\rho \in \mathfrak{U}$ and some integer $n, \Sigma_{i=1}^{\infty} i \mu_{i}\left(m_{\alpha}\right)<n$ for $\alpha>\rho$. Since $\bigcup_{i=1}^{\infty} \lambda_{i}^{-1}\{1\} \supset$ $\bigcup_{i=1}^{\infty} W_{i} \supset M$ and $\mu_{i} \geqslant \lambda_{i}$, it now follows that $\left\{m_{\alpha} \mid \alpha>\rho\right\} \subset \lambda_{1}^{-1}\{1\} \cup \ldots$ $\cup \lambda_{n}^{-1}\{1\}$. It follows that for some $k \leqslant n$ and some cofinal $\mathfrak{D} \subset \mathfrak{A},\left\{m_{\alpha} \mid \alpha \in \mathfrak{D}\right\}$ $\subset \lambda_{k}^{-1}\{1\}$. Since $\left\{f\left(m_{\alpha}\right) \mid \alpha \in \mathfrak{D}\right\}$ converges to $y$, we then have $\left\{\phi_{k}\left(m_{\alpha}\right) \mid \alpha \in \mathfrak{D}\right\}$ converging to $y_{2 k}$. But on $\lambda_{k}^{-1}\{1\}, \phi_{k}$ is a closed $b^{*}$-embedding. It follows that $y_{2 k}=\phi_{k}(m)$, some $m \in \lambda_{k}^{-1}\{1\} \cap A$, and $\left\{m_{\alpha} \mid \alpha \in \mathfrak{D}\right\}$ converges to $m$ in $M\left(b^{*}\right)$. The continuity of $f$ then assures us that $\left\{f\left(m_{\alpha}\right) \mid \alpha \in \mathfrak{D}\right\}$, and hence $\left\{f\left(m_{\alpha}\right) \mid \alpha \in\right.$ थै , converges to $f(m)$ in $B^{*}\left(b^{*}\right)$ so that $y=f(m)$. Thus $f(A)$ is closed in $\Sigma B^{*}\left(b^{*}\right)$, and we have shown that $f: M\left(b^{*}\right) \rightarrow \Sigma B^{*}\left(b^{*}\right)$ is a closed embedding. This completes the proof that $f: M \rightarrow \Sigma B^{*}$ is a closed $\left(C^{p}, b^{*}\right)$-embedding.

Now suppose $\chi: \Sigma B^{*} \rightarrow E^{*}$ is a $\left(\tau^{*}, b^{*}\right)$-Banach completion. Let $m \in M$. Since $f: M \rightarrow \Sigma B^{*}$ is a closed $\left(C^{p}, b^{*}\right)$-embedding there is a $b^{*}$-neighborhood $V$ of $m$ in $M$ and an integer $n$ such that $f(V) \subset\left(B^{*}\right)^{n}$ and $f: V \rightarrow\left(B^{*}\right)^{n}$ is a split $\left(C^{p}, b^{*}\right)$-embedding. But $\chi /\left(B^{*}\right)^{n}$ is a linear split $\left(C^{p}, b^{*}\right)$-embedding, so $(\chi \circ f) / V$ is a split $\left(C^{p}, b^{*}\right)$-embedding into $E^{*}$. Thus, $\chi \circ f: M \rightarrow E^{*}$ is a local split $\left(C^{p}, b^{*}\right)$-embedding. 
By V. $1, \chi \circ f$ will be a closed split $\left(C^{p}, b^{*}\right)$-embedding if $\chi \circ f: M\left(b^{*}\right) \rightarrow$ $E^{*}\left(b^{*}\right)$ is a closed topological embedding. In order to show this let $A$ be closed in $M\left(b^{*}\right)$, and let $\left\{m_{\alpha} \mid \alpha \in \mathscr{Q}\right\}$ be a net in $A$ such that $\left\{\chi \circ f\left(m_{\alpha}\right)\right\}$ converges to $y \in E^{*}$. Let $f_{1}\left(m_{\alpha}\right)=\Sigma_{i=1}^{\infty} i \mu_{i}\left(m_{\alpha}\right) a^{*}$ and let $\pi: E^{*} \rightarrow \chi\left(\left(B^{*}\right)^{1}\right)$ be the natural projection, where $E^{*}=\chi\left(\left(B^{*}\right)^{1}\right) \oplus_{1} E^{*}$. Since $\chi\left({ }_{1} B^{*}\right) \subset{ }_{1} E^{*}$ we obtain

$$
\left(\left(\chi /\left(B^{*}\right)^{1}\right)^{-1} \circ \pi \circ \chi \circ f\right)\left(m_{\alpha}\right)=f_{1}\left(m_{\alpha}\right) .
$$

Hence

$$
f_{1}\left(m_{\alpha}\right) \rightarrow\left(\left(\chi /\left(B^{*}\right)^{1}\right)^{-1} \circ \pi\right)(y)=\gamma a^{*} \quad \text { some } \gamma \geqslant 0 .
$$

Thus, there is a $\rho \in \mathscr{U}$ and an integer $n$ such that $\mu_{i}\left(m_{\alpha}\right)<1$, and hence $\lambda_{i}\left(m_{\alpha}\right)$ $=0$, for $\alpha>\rho$ and $i>n$. Therefore, $f\left(m_{\alpha}\right) \in f(A) \cap\left(B^{*}\right)^{2 n+1}$ if $\alpha>\rho$. Since $\chi /\left(B^{*}\right)^{2 n+1}$ is a closed embedding it now follows that $y \in(\chi \circ f)(A)$. We have shown that $\chi \circ f$ is a closed split $\left(C^{p}, b^{*}\right)$-embedding. This completes the proof of Theorem V. 5 (and hence also Theorem II. 3).

VI. Remark. Given a differentiable map $g: B^{*} \rightarrow E^{*}$ and $x^{*} \in B^{*}$, let $D g\left(x^{*}\right): B^{*} \rightarrow E^{*}$ be the derivative of $g$ at $x^{*}$. Note that the $\left(C^{\infty}, b^{*}\right)$-morphism $\phi: B^{*} \rightarrow R$ constructed in the proof of Lemma IV. 1 satisfies the property that $D \phi\left(x^{*}\right): B^{*} \rightarrow R$ is also a $\left(C^{\infty}, b^{*}\right)$-morphism for each $x^{*} \in B^{*}$. This is because for each $r>0$ there is an integer $n$ such that $\phi / B_{r}^{*}=\prod_{i=1}^{n} \widetilde{\phi}_{i} \circ \hat{x}_{i}\left(\widetilde{\phi}_{i}\right.$ and $\hat{x}_{i}$ as defined in the proof of IV. 1) and each

$$
D\left(\tilde{\phi}_{i} \circ \hat{x}_{i}\right)\left(x^{*}\right)=D \widetilde{\phi}_{i}\left(x^{*}\left(x_{i}\right)\right) \circ \hat{x}_{i}: B^{*}\left(b^{*}\right) \rightarrow R
$$

is continuous. Similar reasoning establishes that the $\lambda$ constructed in Proposition IV. 2 also satisfies the property that $D \lambda\left(x^{*}\right): B^{*} \rightarrow R$ is a $\left(C^{\infty}, b^{*}\right)$-morphism for each $x^{*} \in B^{*}$.

Now define a $S\left(\right.$ trong)-( $\left(C^{p}, b^{*}\right)$-manifold $M$ by requiring that $M$ be a $\left(C^{p}, b^{*}\right)$-manifold such that the transition maps $\phi_{j} \phi_{i}^{-1}$ also satisfy the requirement that $D\left(\phi_{j} \phi_{i}^{-1}\right)\left(x^{*}\right): B_{i}^{*}\left(b^{*}\right) \rightarrow B_{j}^{*}\left(b^{*}\right)$ be continuous for each $x^{*} \in B_{i}^{*}$. Similarly define $S$-( $\left.C^{p}, b^{*}\right)$-morphisms, etc. We have just indicated that the $\lambda$ constructed in Proposition IV. 2 is an $S$-( $\left.C^{\infty}, b^{*}\right)$-morphism. Using this, the proofs given in this paper yield analogous theorems about $S$ - $\left(C^{p}, b^{*}\right)$-manifolds.

In reference to the remark at the end of §II, observe that there is no distinction between $\left(C^{p}, b\right)$-manifolds and $S-\left(C^{p}, b\right)$-manifolds. This is because any linear map between Banach spaces which is continuous with respect to the metric topology is automatically continuous with respect to the weak, and hence also bounded weak, topology (cf. Proposition III. 2). 


\section{BIBLIOGRAPHY}

1. R. A. Bonic and J. Frampton, Smooth functions on Banach manifolds, J. Math. Mech. 15 (1966), 877-898. MR 33 \#6647.

2. J. Dieudonné, Natural homeomorphisms in Banach spaces, Proc. Amer. Math. Soc. 1 (1950), 54-59. MR 11, 524.

3. N. Dunford and J. T. Schwartz, Linear operators. I: General theory, Pure and Appl. Math., vol. 7, Interscience, New York, 1958; 2nd printing, Interscience, New York, 1964. MR 22 \#8302.

4. James Eells, Jr., A setting for global analysis, Bull. Amer. Math. Soc. 72 (1966), 751-807. MR 34 \#3590.

5. R. A. Graff, Elements of local non-linear functional analysis, Thesis, Princeton University, 1972.

6. D. W. Henderson, Stable classification of infinite-dimensional manifolds by homotopy-type, Invent. Math. 12 (1971), 48-56. MR 44 \#7594.

7. N. H. Kuiper and B. Terpstra-Keppler, Differentiable closed embeddings of Banach manifolds, Essays on Topology and Related Topics (Mémoires dédiés à Georges de Rham), Springer, New York, 1970, pp. 118-125. MR 41 \#9300.

8. J. Kurzweil, On approximation in real Banach spaces, Studia Math. 14 (1954), 214-231 (1955). MR 16, 932.

9. K. Morita, Star-finite coverings and the star-finite property, Math. Japon. 1 (1948), 60-68. MR 10, 204.

10. R. S. Palais, Banach manifolds of fiber bundle sections, Actes Congr. Internat. Math., Nice, 1970, vol. 2, Gauthier-Villars, Paris, 1971, pp. 243-249.

11. W. Rudin, Real and complex analysis, McGraw-Hill, New York, 1966. MR 35 \#1420.

DEPARTMENT OF MATHEMATICS, CORNELL UNIVERSITY, ITHACA, NEW YORK 14850

Current address. Department of Mathematics, Vanderbilt University, Nashville, Tennessee 37235 